\title{
Influence of humic substances on the decomposition of insecticides in rice fields of Bali Island
}

Gasanov M.E. ${ }^{1}$, Zavgorodnyaya Yu.A. ${ }^{2}$

${ }^{1}$ Skoltech CDISE, Moscow, Russia, gasanov.msu@yandex.ru

${ }^{2}$ Lomonosov MSU, Moscow, Russia

doi: 10.36291/HIT.2019.gasanov.071

The need to intensify agriculture in densely populated areas of South-East Asia leads to the active use of fertilizers and pesticides. For pest management of rice farmers often use insecticides of the group of neonicotinoids. It is known that humic substances affect the behavior of organic pollutants in the environment. Humic substances are able to bind toxicants, changing their migration ability, as well as to have a stimulating effect on the microbial community of the soil, accelerating the process of decomposition of organic pollutants.

The aim of our work was to assess the effect of humic product on the transformation and migration of pesticides in soils. The object of the study was widely used on the island of Bali drugs based on neonicotinoid insecticides: thiamethoxam, Imidacloprid sand fipronil. During the model experiment, soil samples with insecticides and humic preparation were incubated for $14,28,56$ days at a temperature of $25^{\circ} \mathrm{C}$. Humic preparation was introduced as a solution with a concentration of $0.25 \%$ ( $5 \mathrm{ml}$ per $25 \mathrm{~g}$ of soil). During the experiment, the indicators of the microbial state of the soil (basal respiration, nitrogen fixation, methane formation) were measured. The method for determining pesticides in soil is based on acetonitrile extraction and subsequent determination on a chromato-mass spectrometer (HPLC-Q-TOF).

The results show an increase in methane formation in the soil on day 7 , which can be associated with a significant number of methanogenic bacteria in flooded rice cakes. As a result of the experiment, after 14 days, a decrease in the content of Imidacloprid in the variant with the introduction of humic substances by $30 \%$ was revealed. The decrease in the detectable pesticide in soil may be due to the specific effect of humic preparation on certain groups of microorganisms. 